\title{
Barium Retention Following Examination for Pylorus Stenosis
}

\author{
Michal Slastan ${ }^{\mathrm{a}, \mathrm{b}}$, Dusan Dobrota ${ }^{\mathrm{a}}$
}

\begin{abstract}
Barium retention is a rare complication of upper gastrointestinal tract examination when using barium sulphate as a contrast agent. We present a case of rare barium retention and precipitation in the stomach in terrain of chronic gastrostasis and the review of literature on complications of barium sulphate administration. A 38-year-old patient was diagnosed with benign pylorostenosis. After consulting radiology department barium contrast-enhanced X-ray has been indicated. The diagnostic process and the resulting treatment of the complications are described. Patient's informed consent was obtained. The use of barium sulphate as a contrast agent led to stasis and impaction of the contrast agent, which led to emergency surgery. Histology proved malignant stenosis of the pylorus. The resection edges proved insufficient and a repeated resection was performed. At the time of submission the patient has no confirmed metastatic lesions. In cases of suspected gastrostasis and serious stenosis of the intestine, use of barium sulphate should be avoided and iodine-based contrast agents should be used instead.
\end{abstract}

Keywords: Barium; Swallow; Gastrostasis; Pylorostenosis

\section{Introduction}

The examination using barium contrast agent was pioneered by Walter Cannon in the year 1902, however widespread use of barium as a contrast agent came only during the first world war when barium replaced bismuth as a go to agent, since bismuth was necessary for the war industry. Barium sulphate with its advantageous properties for both upper and lower gastrointestinal tract examinations became irreplaceable and is used to this day. The use of barium contrast agents is generally considered safe; however there are risks tied to the application of barium. One of the risks is aspiration of the contrast agent; an-

Manuscript submitted May 13, 2019, accepted June 10, 2019

aDepartment of Medical Biochemistry, Jessenius Medical Faculty in Martin, Comenius University in Bratislava, Bratislava, Slovakia

${ }^{b}$ Corresponding Author: Michal Slast'an, Department of Medical Biochemistry, Jessenius Medical Faculty in Martin, Comenius University in Bratislava, Bratislava, Slovakia. Email: michalslastan20@gmail.com

doi: https://doi.org/10.14740/gr1191 other risk is leakage of the contrast agent into mediastinum or intraperitoneally in the case of the perforation of the digestive tract. These complications often lead to inflammation, which may or may not be life-threatening. Barium aspiration may not always lead to pneumonia, but in the elderly and in case of preexisting lung disease may present with a severe inflammation, acute respiratory failure and be life-threatening [1]. In case of barium leakage into the peritoneal cavity or into mediastinal spaces the ensuing complications can severely endanger the survival of the patient with surgical removal of the contrast agent through surgical techniques and lavage difficult, much more so when compared with the removal of a water soluble contrast agent based on iodine [2]. Cases of barium sulphate precipitation and sedimentation in the gastrointestinal tract are rare; however complications from barium impaction in the intestines almost always require open surgery to resolve [3]. It has been reported on the sedimentation of barium contrast agent in the appendix; however the contrast agent is usually eliminated from this location within $72 \mathrm{~h}$. There have however been cases described with a long-term impaction of barium in this location and subsequent inflammations requiring surgical resection of the appendix [4]. The use of barium as a contrast agent is less and less common. In select cases it is still the best approach at verifying and imaging the pathologic anatomy [5]. Aside from the use as a contrast medium there have been cases described where barium sulphate has been used to stop bleeding from inflammated diverticula of the colon in the form of a high concentration enema. Such barium application leads to hemostasis and the patient avoids more invasive surgical procedures [6]. This case report describes rare complications caused by the precipitation and stasis of the contrast agent in the stomach due to pylorostenosis.

\section{Case Report}

A 38-year-old patient arrived at the surgical department complaining of long-term problems with food intake. Patient history showed previous surgery for congenital pylorostenosis in childhood. The patient was examined at the surgeon's office and the surgeon ordered a native X-ray and ultrasound examination; both exams showed no pathology. A computed tomography (CT) scan was performed (Fig. 1) which raised the suspicion of gastrostasis. With the suspicion of recurrent pylorostenosis a radiologist was consulted and a contrastenhanced examination of the upper gastrointestinal tract was indicated. Radiologist elected to use barium sulphate as the 


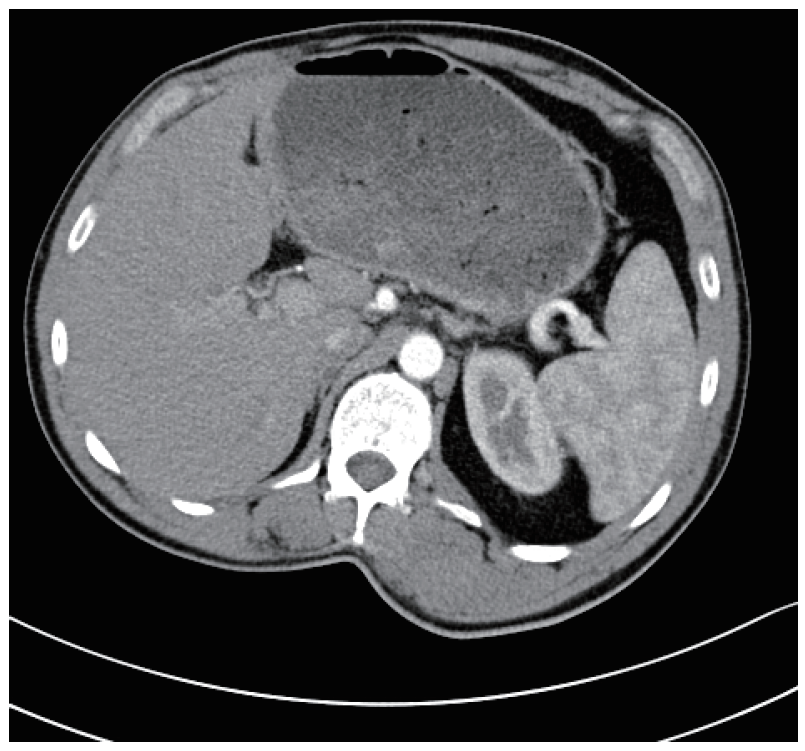

Figure 1. CT scan demonstrates gastrostasis.

contrast agent. The examination demonstrated stenosis of the pylorus with gastrostasis which was confirmed in a repeated $\mathrm{X}$-ray after $30 \mathrm{~min}$ with stasis of all the contrast agent in the stomach (Figs. 2, 3). The surgeon suspected a benign stenosis of the pylorus and scheduled the patient for planned surgical intervention. In 5 days the patient made a unplanned return to

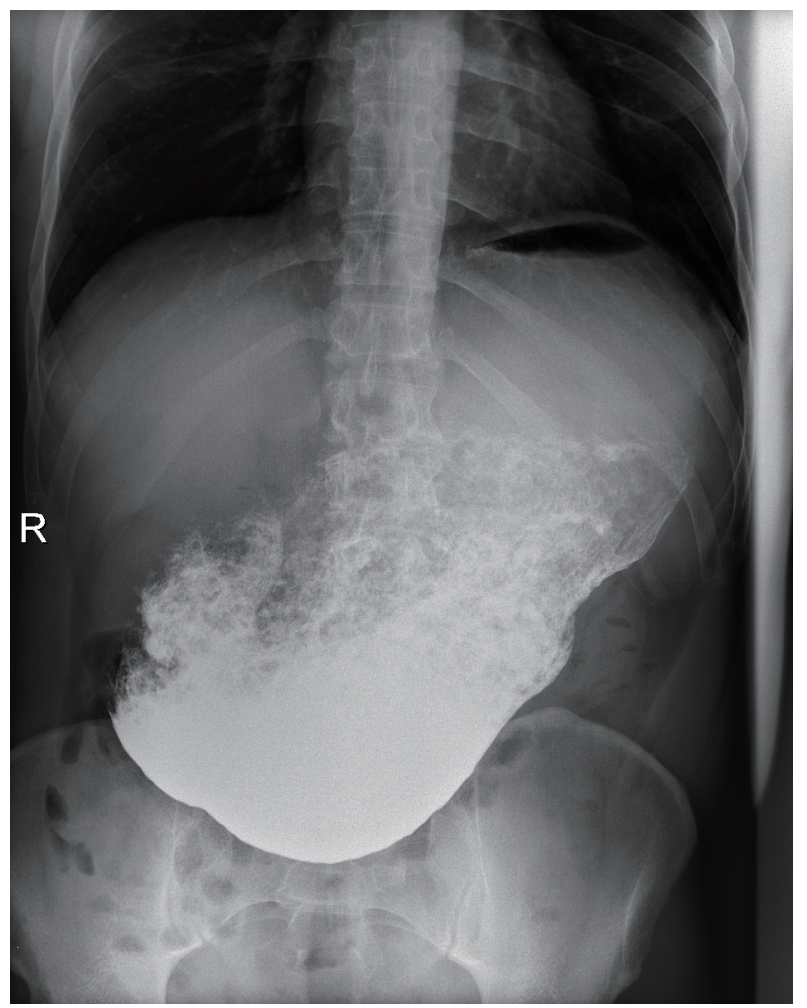

Figure 2. X-ray examination: barium sulphate was used as contrast agent. Repeated examination 30 min after barium administration demonstrates gastrostasis.

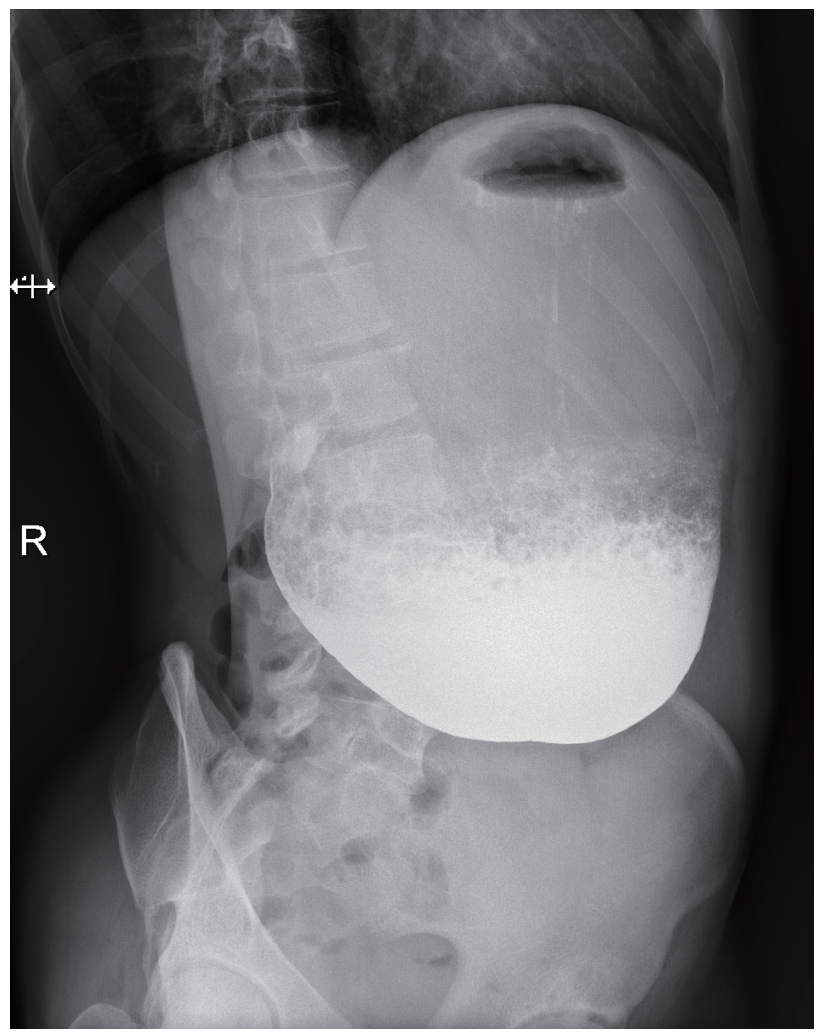

Figure 3. X-ray examination: $30 \mathrm{~min}$ after barium administration, lateral oblique projection.

the surgeon's office complaining of substantial and increasing pain in left epigastrium. The left epigastric region was tender and considerable pain was felt on palpation. The surgeon ordered an X-ray examination that displayed evidence of the stasis of a large amount of contrast agent in the stomach (Fig. 4). Inflammation parameters were not elevated (C-reactive protein (CRP) $0.3 \mathrm{mg} / \mathrm{L}$, procalcitonine $0.086 \mu \mathrm{g} / \mathrm{L})$. The patient was admitted to the surgery ward. Nasogastric tube was inserted with an unsuccessful attempt to evacuate the contrast agent. During hospitalization the patient's condition deteriorated and an immediate laparotomy was indicated to find a surgical solution to the patient's condition. During surgical intervention a tumorous lesion was identified on the pylorus with the diameter of $5 \mathrm{~cm}$, dilated stomach and adhesions. After mobilizing the stomach and duodenum a large amount of precipitated and sedimented barium sulphate was carefully scooped out with emphasis on preventing its intraperitoneal spillage. A BII partial stomach resection was performed, the removed tissue was send to pathology department for histological examination. Roux-en-Y gastrojejunal anastomosis was established, a repeated lavage of the peritoneal cavity was carried out. Tygon tube 27 drainage was inserted near the stump of the duodenum and into the rectovesical excavation. After surgical intervention the patient was moved to the central intensive care unit. Intense antibiotics therapy was initiated, patient received infusions and nutritional support. On the second day following surgery patient's condition worsened with the elevation of inflammation markers (CRP $78.9 \mathrm{mg} / \mathrm{L}$, 


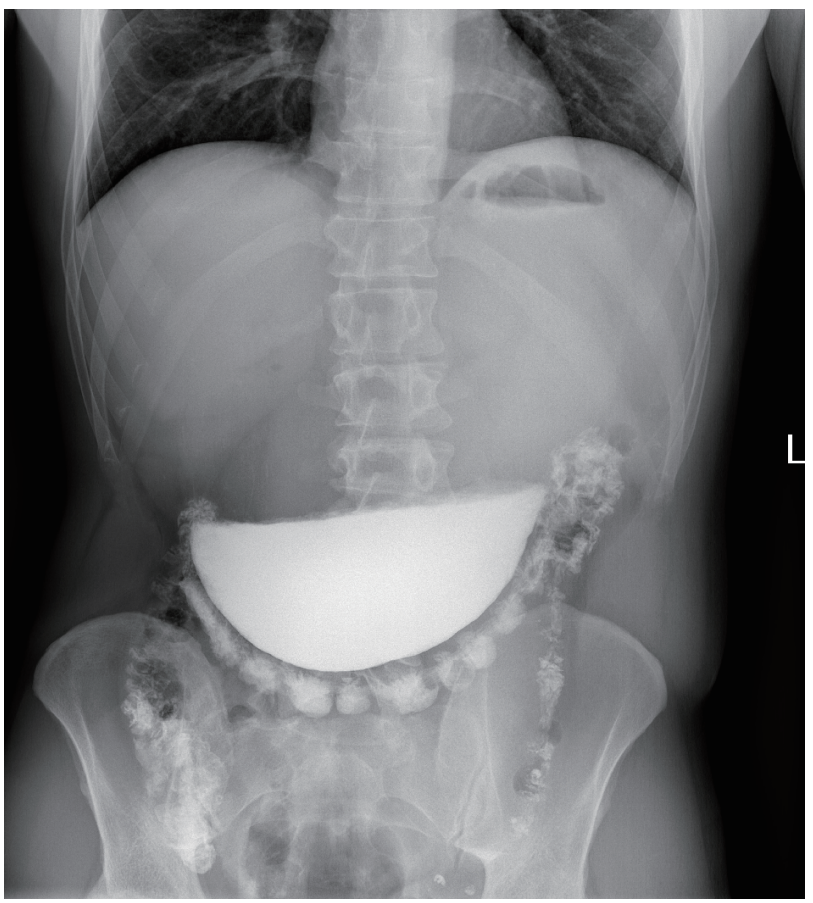

Figure 4. X-ray examination demonstrates stasis of the contrast agent, minimal amount of contrast agent passed through the pylorus.

procalcitonine $4.45 \mu \mathrm{g} / \mathrm{L})$. Leak of the anastomosis or contrast agent spillage with peritonitis was suspected. A CT scan was indicated, however identified no contrast agent spillage intraperitoneally and no anastomosis failure. Subsequently an ampule of methylene blue in $100 \mathrm{~mL}$ of physiological solution was administered through the nasogastric tube, the drained exudate was observed for signs of methylene blue, but none was found. The drainage bags contained $200 \mathrm{~mL}$ of sanguinolent fluid with no hint of methylene blue. In the morning of the third day following surgery another CT exam was indicated

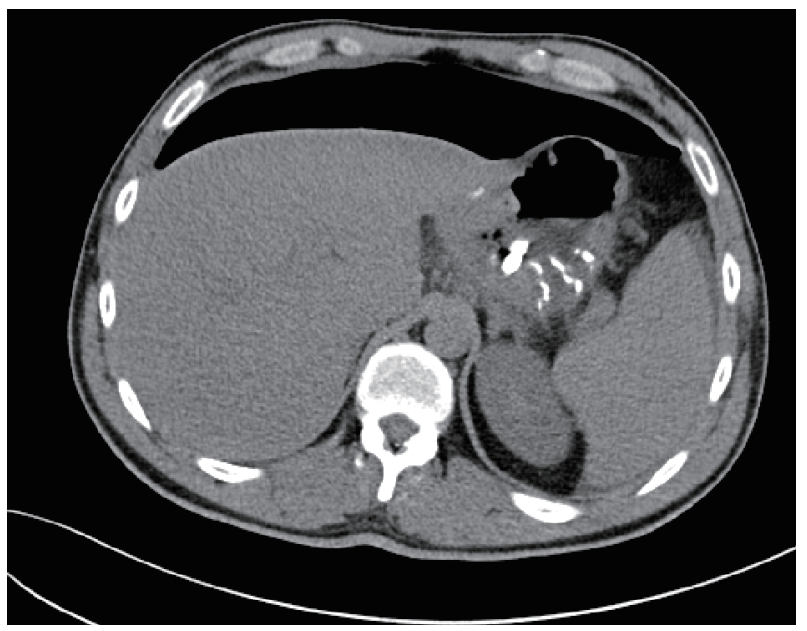

Figure 5. CT examination after partial resection of the stomach, the examination demonstrated only minimal amount of contrast agent intraperitoneally outside the gastrointestinal tract; and an active leak was not identified.

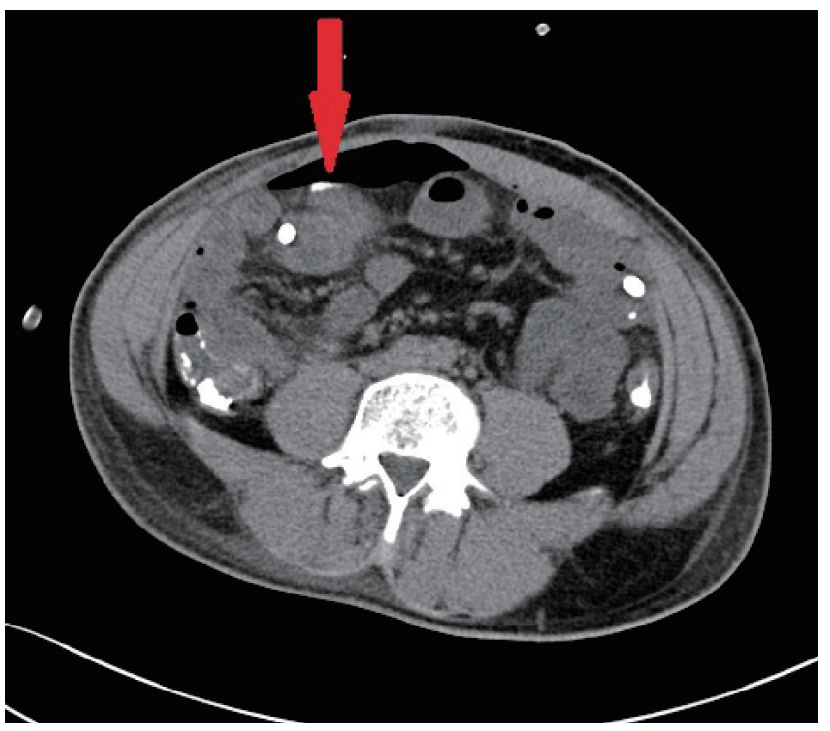

Figure 6. CT examination after partial resection of the stomach, small amount of contrast agent intraperitoneally outside the digestive tract is marked by the arrow.

(Figs. 5, 6), and a small amount of contrast agent was identified in the paragastric intraperitoneal region. The previous CT exam was reviewed and the same amount of contrast agent was identified next to the stomach, active leak was therefore not suspected. Laboratory results were however worsening with increase in inflammation parameters (CRP $267.60 \mathrm{mg} / \mathrm{L}$, procalcitonine $25.90 \mu \mathrm{g} / \mathrm{L})$. Sepsis was suspected, but the patient remained afebrile. Due to anastomosis dehiscence being repeatedly unconfirmed on $\mathrm{CT}$ exams, considered surgical revision was not performed. Antibiotic therapy was revised and vasopressor support was introduced. On the fourth day following surgery patient's condition improved, therapy with antibiotics and vasopressors continued, and the amount of exudate drained was significantly lower. The surgical wound was found to be dehiscent and phlegmonous, local therapy was applied. A week after the surgery patient's condition was improving further with stools being passed. Vasopressors were discontinued. Inflammation parameters improved considerably (CRP $78.60 \mathrm{mg} / \mathrm{L}$, procalcitonine $2.85 \mu \mathrm{g} / \mathrm{L}$ ). Two weeks after the surgery with further improvement of inflammation parameters (CRP $13.80 \mathrm{mg} / \mathrm{L}$ ) the patient was discharged. Histological exam of the resected tissue has confirmed the diagnosis of malignant stenosis of the pylorus (moderately differentiated adenocarcinoma) and the resection margin was positive for carcinoma. A repeated resection with the revision of the anastomosis was indicated. The surgical wound again healed per secundam. The patient was referred to the department of clinical oncology where chemotherapy was initiated. The last positron emission tomography (PET)/CT exam from July 9, 2018 found no metastatic lesions.

\section{Discussion}

The application of barium contrast agent in upper gastrointes- 
tinal tract examinations is generally considered safe, and barium sulphate is considered inert. The experience with barium sulphate in our institution mirrors this claim, for this was the first case of severe complication following barium sulphate administration, which required surgery in the last decade. The frequency of barium use is nonetheless decreasing in our institution. In modern times it is increasingly replaced by methods of endoscopy and other imaging methods, in spite of the fact that patients are afraid of endoscopic examinations and there are risks of complications as well. Well known risks associated with barium sulphate use are aspiration of the contrast agent, leakage of the contrast agent through preexisting fistulae or in case of perforation of the gastrointestinal tract. Less known is the risk of barium contrast agent retention in cases of stenosis or obturation of the digestive tract, functional disorders of the gastrointestinal tract and diverticula. Aspiration of barium sulphate is not considered particularly dangerous and barium behaves as an inert foreign body, there are however reported cases of severe acute respiratory insufficiency after barium aspiration [7]. Bronchoscopy and lavage are concededly indicated only in cases of manifest complications with respiratory insufficiency. In case of upper gastrointestinal tract perforation leakage of gastric contents along with barium contrast agent intraperitoneally causes chemical peritonitis with exudation of a large volume of fluid and the creation of adhesions. In case of lower gastrointestinal tract perforation fecal bowel contents and barium contrast agent leak intraperitoneally or retroperitoneally. Intraperitoneal leakage from lower gastrointestinal tract leads to much more severe peritonitis with the need for volumotherapy and early application of antibiotics. In both cases immediate laparotomy with peritoneal toilet and the extraction of the highest possible volume of the leaked material are recommended. If the volume of leaked barium sulphate is high ileostomy should be considered. Omentum covered in barium contrast agent is considered an indication for its resection. In most cases nutritional support is necessary $[8,9]$. As mentioned, the application of barium sulphate is generally safe, however rare complications tend to be severe and, their treatment difficult. Although considered chemically inert, barium sulphate leakage intraperitoneally, retroperitoneally and into the mediastinum as well as aspiration and impaction leads to severe inflammation, which almost always requires surgical intervention. The complication suffered by our patient is one of the rarest, the stasis and precipitation of barium contrast led to an urgent surgical intervention, during which the evacuation of the contrast agent proved a challenge, and a small amount of contrast agent has leaked into the peritoneal cavity. Due to careful lavage of the peritoneal cavity the resulting inflammation was only mild. The complications could have been avoided altogether by choosing iodine-based contrast agent. Even though the properties (viscosity, adhesiveness, high attenuation of X-rays, lack of absorption and lack of toxicity) of barium sulphate are much better when compared with iodine-based contrast agents for upper gastrointestinal tract examinations, its use must be carefully considered in the terrain of suspected gastrostasis or the stenosis and obturation of gastrointestinal tract. If one of these is suspected iodine-based contrast agent are a safer alternative and should be used instead.

\section{Conclusions}

The use of barium sulphate as a contrast agent in upper gastrointestinal tract is safe with only rare incidence of complications. Barium sulphate is generally inert, and allergic reactions are very rare. It can however cause serious complications once it leaks outside the gastrointestinal tract, in case of perforation and aspiration. Dangerous complications can be caused by retention of the contrast agent, its precipitation and impaction. Therefore application of the contrast agent must be carefully considered in situations where retention is likely. Because of the gastrostasis suspected on the CT examination, electing barium sulphate as the contrast agent in our patient proved to be a bad decision. Iodine-based contrast agent should be used for the upper gastrointestinal tract examinations in cases of suspected gastrostasis and severe stenosis or obturation of the intestines.

\section{Acknowledgments}

I would like to thank my tutor and mentor Professor Dusan Dobrota for all his help and good advice.

\section{Financial Disclosure}

The study received no funding, and did not receive any specific grant from any funding agency in the public, commercial, or not-for-profit sectors.

\section{Informed Consent}

Patient's informed consent was obtained.

\section{Conflict of Interest}

The authors declare that they have no conflict of interest.

\section{Author Contributions}

Michal Slast'an substantially contributed to the conception, design of the work, acquired the data, contributed to analysis and interpretation, drafted the work, approved the final version to be published, and agreed to be accountable for all aspects of the work. Dusan Dobrota substantially contributed to the interpretation of data, revised the work for content, approved the published version, and agreed to be accountable for all aspects of the work.

\section{References}

1. Kawsar HI, Shahnewaz J, Ricaurte B, Daw HA. Barium 
aspiration. BMJ Case Rep. 2012;2012:bcr0220125891.

2. Ruth Ann Ehrlich, Dawn M Coakes. LIC - Patient care in radiography, with an introduction to medical imaging.

3. Kurer MA, Davey C, Chintapatla S. Intestinal obstruction from inspissated barium (Barolith): a systematic review of all cases from 1950 to 2006. Colorectal Dis. 2008;10(5):431-439.

4. Katagiri H, Lefor AK, Kubota T, Mizokami K. Barium appendicitis: A single institution review in Japan. World J Gastrointest Surg. 2016;8(9):651-655.

5. Mahajan A, Desai S, Sable NP, Thakur MH. Status of barium studies in the present era of oncology: Are they a history? Indian J Med Paediatr Oncol. 2016;37(4):223226.

6. Niikura R, Nagata N, Yamano K, Shimbo T, Uemura N.
High-dose barium impaction therapy is useful for the initial hemostasis and for preventing the recurrence of colonic diverticular bleeding unresponsive to endoscopic clipping. Case Rep Gastrointest Med. 2013;2013:365954.

7. Hundemer GL, Kumar V, Vaduganathan M. Largevolume barium aspiration. Proc (Bayl Univ Med Cent). 2015;28(2):183-184.

8. Karanikas ID, Kakoulidis DD, Gouvas ZT, Hartley JE, Koundourakis SS. Barium peritonitis: a rare complication of upper gastrointestinal contrast investigation. Postgrad Med J. 1997;73(859):297-298.

9. Vahedian-Ardakani J, Nazerani S, Saraee A, Sarmast A, Saraee E, Keramati MR. Proper management for morbid iatrogenic retroperitoneal barium insufflation. Ann Coloproctol. 2014;30(6):285-289. 